\title{
Fundamental Role of Local Curvature of Crystal Structure in Plastic Deformation and Fracture of Solids
}

\author{
V. E. Panin ${ }^{1,2, \text { a) }}$ and V. E. Egorushkin ${ }^{1}$ \\ ${ }^{1}$ Institute of Strength Physical and Materials Science SB RAS, Tomsk, 634055, Russia \\ ${ }^{2}$ National Research Tomsk Polytechnic University, Tomsk, 634050, Russia

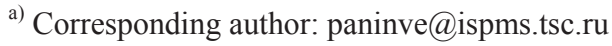

\begin{abstract}
It is shown that strain-induced defects of all kinds are nucleated in local curvature zones of the interfaces between 3D-crystals and 2D-planar subsystem similar to a laser pumping mechanism. Mobile curvature solitons are generalized wave structural carriers of plastic flow. Fracture is the structural phase decay of a crystal in the zones of very high curvature.
\end{abstract}

Keywords: strain-induced defects, cracks, curvature, solitons, waves, plastic flow, fracture

\section{INTRODUCTION}

Physical mesomechanics treats a deformable solid as a nonlinear multiscale hierarchically organized system. Particular attention is given to nonlinear behavior of a solid in any external field. This nonlinearity problem is impossible to solve if we reason from the steady structure of a translation-invariant crystal. Any strain-induced defect is nucleated within local crystal curvature, in the zone of which local structural transformation occurs as a strictly nonlinear process. Therefore, the nonlinearity problem is inevitably related to the mechanisms of local structural transformations in local curvature zones as nonequilibrium thermodynamic processes. It is these issues that we consider in the paper.

\section{NUCLEATION OF STRAIN-INDUCED DEFECTS IN SOLIDS BY LASER PUMPING MECHANISM}

The multiscale description of a deformable solid as a nonlinear hierarchically organized system is managed to solve the problem of nucleation of strain-induced defects. According to $[1,2]$ a deformable solid should be considered to consist of two subsystems: a 3D-translation-invariant crystal and a 2D-planar subsystem comprising surface layers and all internal interfaces. A 2D-planar subsystem is characterized by a cluster structure and absence of translation invariance [3]. Primary plastic shears in a solid under loading are developed within a planar subsystem as nonlinear waves of structural transformations (see below). The distribution of normal and shear stresses at the interface between 3D-crystalline and 2D-planar subsystems is periodical [4]. As a consequence, nonlinear plastic shears within a planar subsystem make up the concentration fluctuation of ions localized in zones of tensile normal stresses (the Bose condensate [3]) (Fig. 1). Such ion clusters are screened by electrons from neighbour atoms. It causes the formation of very high local curvature within the adjacent crystalline substrate where the new allowed structural states appear in interstitial spaces.

It is very important that a new electron fluctuation band below the Fermi level appears in the electron spectrum corresponding to the new structural states in interstitial space. In these conditions high excited ions and electrons in planar clusters are to be transferred to new low-energy states in interstitial space as a strain-induced defect in a crystal. It is the general mechanism of any

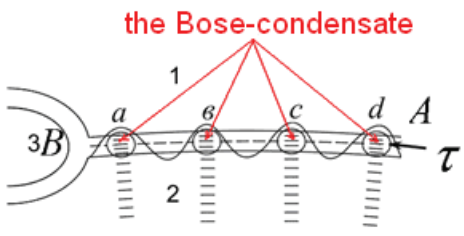

FIGURE 1. Laser pumping mechanism of the Bosecondensate formation in the $a, b, c, d$ zones of the planar subsystem $A B$ which act as the sources of dislocation generation in a $3 \mathrm{D}$-crystal 
strain-induced defect nucleation similar to laser pumping. If there is no electron fluctuation band in a solid electron spectrum related to the curvatured interstitial space, the development of local curvature causes brittle fracture.

The kind of a strain-induced defect nucleated by the laser pumping mechanism depends upon the scale of local curvature related to fluctuation of ion concentration in a 2D-planar subsystem. In zones of a small curvature scale, dislocations nucleate. If the curvature scales are middle, a crystal under loading generates shear bands which are translation-rotation strain-induced defects. They transform elastic curvature of a solid into plastically misoriented one [5]. Very extended curvature in a crystal under loading can be relaxed by the disclination nucleation. The new kind of nonequilibrium vacancies named as curvature vacancies plays dominant role in this process. They allow forming a disclination as a strain-induced defect of high misorientation.

At last for very large local curvature scales, the number of curvature vacancies becomes so high that they form a crack and initiate fracture. This process is classified as the structural phase decay of a loaded solid [6].

\section{MOBILE CURVATURE SOLITONS AS GENERALIZED WAVE STRUCTURAL CARRIERS OF PLASTIC FLOW}

Any kind of localized plastic flow is related to the motion of the definite curvature soliton. It will be shown for localized plastic flow within a noncrystallograhyic 2D-planar subsystem and for the crystallograhyic motion of dislocations.

Let us consider a localized defect flow in a planar structure when the plastic deformation along the direction $L$ is channeled between two layers of an elastically deformed material. The general coordinate system is chosen so that the axis $z$ is directed along $L$, and $x$ and $y$ vary within the plastically deformed layer thickness. According to [7], the distribution of plastic flow in a local $(r<L)$ region has the form:

$$
\mathbf{J}=\frac{b_{1}-b_{2}}{4 \pi} \chi(s, t) \mathbf{b}(s, t)(\ln (2 L / r)-1)-\nabla f,
$$

where $\mathbf{b}$ is the binormal vector in the local coordinate system, $\chi$ is the curvature of the region (variation in the curvature of the region axis) due to external load, $t$ is time, $s$ is the current region length, $b_{1}, b_{2}$ are the "Burgers vector" magnitudes for bulk translational and surface or rotational incompatibilities, respectively, $\nabla f$ is the gradient part of the flow due to secondary sources.

The character of channeled spiral propagation of localized plastic deformation in the planar structure is presented in Fig. 2. The spiral curvature $\chi$ is an important parameter of channeled wave propagation of localized plastic flow. Figure 2 shows how this parameter affects the spiral form and the localized transverse strain rate $v$ in the deformed region. It is seen from Fig. 2(a) that with small curvature $\chi$, the transverse strain rate $v$ is low and the spiral experiences weakly pronounced torsion with a large transverse wave length. This pattern is observed for plastic shear in highly nonequilibrium surface layers or in a grain boundary with emition of dislocations. Increasing the curvature causes the transverse wave length to decrease steeply, and the transverse strain rates increase (Fig. 2(b)). With large curvature $\chi$, hydrostatic tension arises in these zones and gives rise to cracks. The foregoing effect is described in detail below: it is shown that any fracture of a solid is its structural phase decay in local hydrostatic tension zones where the curvature $\lambda$ is very high and the Gibbs thermodynamic potential is positive [6].

Another example is related to motion of a dislocation as a curvature soliton polarized in a slip plane. Let us consider the simplest example: a dislocation in a fcc lattice with an hcp core. This means that the planar subsystem, where the dislocation arises, injects each atom in the hcp structural state into the crystal (atom $A$ in Fig. 3). This atom causes "compaction" (closer packing) of the atoms $A-B-E$, and the electronic gas is bound to shield this compaction. The decrease in electronic gas density in the space between the atoms $\mathrm{C}$ and $\mathrm{F}$ causes a local increase in interatomic distance in this space. According to the theory [8], a vacant bifurcation structural state arises in this region.

Under the stress $\tau$, the atom $B$ of the compacted zone in row I turns to the bifurcation state, and the injected atom $A$ takes the place of the atom $B$. In the zone of the atom $A$ in row I, translation invariance is established. Therefore, this process is a relaxation process (a damping factor of the nonlinear wave). However, as the atom $B$ turns to the bifurcation state, the interaction energy in the atomic pairs $B-C$ and $B-F$ increases, and this is an autocatalytic factor of the nonlinear wave.

Next, this nonlinear wave process propagates in a relay-race manner along the slip plane, governing the motion of the polarized curvature soliton under the stress $\tau$. With a split dislocation all atoms of the dislocation core successively experience such zigzag transitions between row I and the row of bifurcation states. Consequently, the motion of dislocations along the slip planes of a translation-invariant crystal should be classified as a nonlinear wave process. 


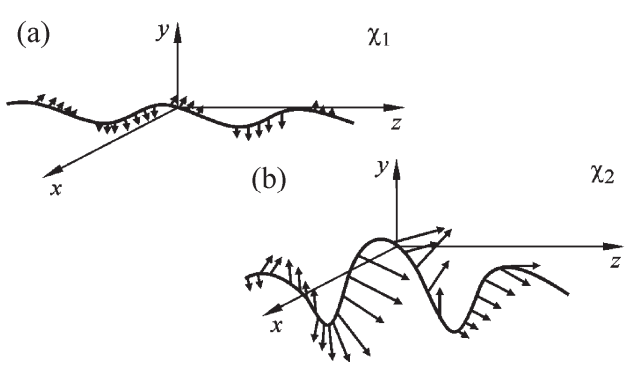

FIGURE 2. The pattern and the rate of localized plastic deformation vs. curvature $\chi$ in the planar subsystem; $\chi_{1}<\chi_{2}$

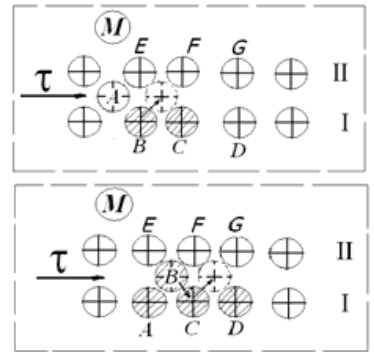

FIGURE 3. The atomic model of a mobile dislocation as a polarized curvature soliton

\section{FRACTURE OF A SOLID AS ITS STRUCTURAL PHASE DECAY IN LOCAL CURVATURE ZONES IN THE VICINITY OOF ZERO GIBBS INTERMODYNAMIC POTENTIAL}

The multiscale approach to plastic deformation and fracture of a solid involves analysis of conjugation and deformation compatibility between its surface layers and its bulk. In a material subjected to alternate bending below the yield strength, most of plastic deformation is concentrated in the surface layers interfacing its elastically loaded substrate. The plastic shear in the surface layer develops in the conjugate directions of maximum tangential stresses. The elastically loaded bulk of the specimen induces couple stresses in plastic shear zones of the surface layer. The formation of rotational deformation modes and high curvature zones in the surface layer gives rise to fatigue surface cracks. High-resolution microscopy allows analyzing the kinetics of this process as structural phase decay of material [9]. Valuable data on the kinetics of structural phase decay of a material at the prefracture stage can be extracted from experiments on titanium with a hydrogenated surface layer subjected to fatigue fracture under alternate bending. In alternate bending, the constrained rotational deformation modes and local curvature zones in surface layers of the material are highly pronounced. The polymorphous transformations occurring in titanium assists the formation of bifurcation structural states in the interstitial space of curvature zones. Hydrogenation of the surface layer increases its molar volume and brings the Gibbs thermodynamic potential close to zero. All the above factors are bound to benefit detection of interface prefracture zones in the hierarchy of rotational deformation modes in titanium with a hydrogenated surface layer under alternate bending. This conclusion is fully confirmed by the research data reported in [9]. Figure 4 shows fragments of the material near the main fatigue crack. The surface layer of the titanium specimen was hydrogenated for $3 \mathrm{~h}$, and this gave rise to high multiscale curvature in the material under alternate bending. Opening of the main crack followed a zigzag pattern with periodic sign reversal of rotations. On the portion represented in Fig. 4, the main crack rotated anticlockwise, initiating the same rotation of the grain conglomerate $O$. According to the angular momentum conservation law at the boundary $K N M L$ of the grain conglomerate $O$ (Fig. 4), a crack of opposite sign (rotating clockwise) is formed.

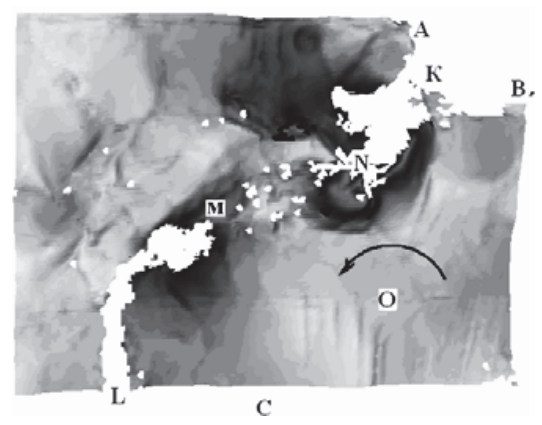

FIGURE 4. Microporosity in the zone $L M N$ and opening of the crack $L M$ by coalescence of micropores; light interferenece profilometer, $\times 450$

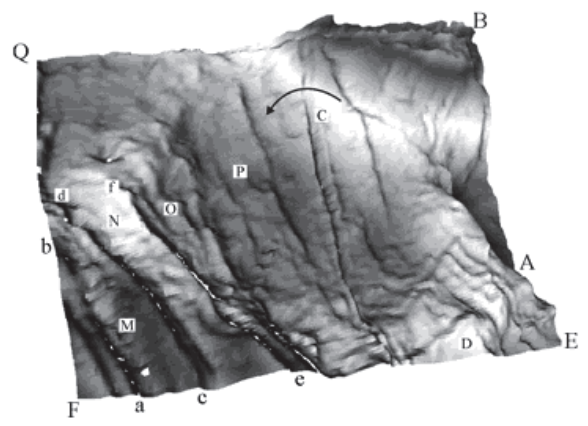

FIGURE 5. Formation of shear-rotation mesobands $M, N, O$, $P$ in the grain $C$ of the hydrogenated surface layer involved in constrained anticlockwise rotation; development of micropores along the high curvature boundaries of the shear-rotation mesobands $a b, c d, e f$; light interference profilometer, $\times 900$ 
In the high curvature zone at the boundary $K N M L$, microporosity has begun developing, which is clearly identified by a New View 6300 interference profilometer. The complex curvature profile at the boundary $K N M L$ (Fig. 4) governed jerky propagation of the accommodation crack $L M N$ while preserving numerous micropores in the extruded material zone $M N$. The portion $K N$ was formed as a fragment of the main crack.

Figure 5 shows another example of microporosity due to rotational deformation of the coarse grain $C$ in the hydrogenated surface layer of the titanium specimen under alternate bending. The grain $C$ experiences anticlockwise rotation initiated by opening of the main crack (the crack is located rightward). The underlying grains $D, E$ in the specimen bulk severely constrains the rotation of the grain $C$. This governs the development of multiscale curvature in the grain $C$ and layerwise delamination (clearly defined in the zones $M, N$ of the grain $C$ ). The delamination develops step by step, forming a sequence of shear-rotation bands $M, N, O, P$, etc. As the grain $C$ rotates, a lamella structure, in which individual lamellae are displaced relative to each other, arises in each band. At the boundaries $a b$, $c d$, ef of conjugate shear-rotation bands, a line porous structure is formed. With small mutual displacements of the bands, the alternation of discrete micropores at their boundaries preserves its periodicity. The periodicity is associated with the chessboard distribution of tensile and compressive normal stresses at the interface of conjugate bands $[10,11]$. With large displacements of the bands, the micropores form extended cracks or get rumpled.

The question as to the formation mechanism of micropores in local curvature zones and their decisive role in crack propagation remains debatable and requires more strict theoretical substantiation. Note that this problem was discussed as applied to intergranular fracture in high-temperature creep and fatigue in which diffusion processes develop intensively. In the present study fatigue fracture developed in titanium at $T=0.2 T_{\mathrm{m}}\left(T_{\mathrm{m}}\right.$ is melting temperature) when the diffusion processes were suppressed, but the curvature effects in the hydrogenated surface layer were highly pronounced.

Under low-temperature deformation, the material undergoes structural phase decay with micropores formation not at the grain boundaries of the polycrystal but at the rotational boundaries where high curvature and tensile normal stresses arise. This conclusion is of great significance because it removes the problem of singularity at the crack tip in linear fracture mechanics [12].

\section{SUMMARY}

Any solid under loading should be considered as consisting of two main subsystems: a 3D-crystal and a 2Dplanar subsystem (surface layers and all interfaces). The primary plastic shears develop within a planar subsystem as nonlinear wave of structural transformations. They form the quasiperiodical distribution of the stress concentration fluctuations of positive ions shielded by electrons. These stress concentrators produce the zones of curvature in adjacent crystal where new allowed structural states appear in interstitial space. The transfer of ions and shielding electrons from a 2D-planar subsystem to the adjacent curvature zone of a 3D-crystal is the nucleation of straininduced defects similar to a laser pumping mechanism. In a 3D-crystal with extended zones of curvature, shear bands propagate transforming an elastic curvature into the plastic one. A shear band generates a crack if the Gibbs thermodynamic potential in a local curvature zone is positive and material undergoes structural phase decay.

The work was supported by Programs of Presidium of the RAS (Nos. 2.2, 8.20, and 25.3), Russian Foundation for Basic Research (No. 14-01-00789), and grant of the President of the Russian Federation for support of leading scientific schools (No. Sh-2817.2014.1).

\section{REFERENCES}

1. V. E. Panin and V. E. Egorushkin, Phys. Mesomech. 14(5-6), 207 (2011).

2. V. E. Panin, V. E. Egorushkin, and A. V. Panin, Physics-Uspekhi. 55, 1260 (2012).

3. V. P. Maslov, Teor. Mat. Fiz. 159(1), 20 (2009).

4. G. P. Cherepanov, J. Appl. Phys. 78, 6826 (1995).

5. V. E. Panin, V. E. Egorushkin, and Yu. I. Pochivalov, "Shear bands as a translation-rotation mode of severe plastic deformation of solids" (in press).

6. V. E. Panin, Proceedings of Europ. Conf. Fracture 19, Kazan, 2012 (Kazan Sci. Center RAS, Kazan, 2012).

7. V. E. Egorushkin, Russ. Phys. J. 35, 316 (1992).

8. M. A. Guzev and A. A. Dmitriev, Phys. Mesomech. 16(4), 285 (2013).

9. V. E. Panin, T. F. Elsukova, and Yu. F. Popkova, Dokl. Akad. Nauk. 453, 155 (2013).

10. V. E. Panin, A. V. Panin, D. D. Moiseenko, et al., Dokl. RAN 409, 606 (2006).

11. I. F. Golovnev, E. I. Golovneva, A. M. Igoshkin, and V. M. Fomin, Phys. Mesomech. 13(5-6) 249 (2010).

12. G. P. Cherepanov, Mechanics of Brittle Fracture (McGraw Hill, New York, 1979. 